\title{
The Effect of Porosity on the Aerodynamic Characteristics of a Rotating Cylinder
}

\author{
Kappas Kussaiynov ${ }^{1}$, Nazgul Kadyralievna Tanasheva ${ }^{1}$, Muratzhan Miryusupovich Turgunov ${ }^{1}$, Guljan \\ Meiramovna Shaimerdenova ${ }^{1} \&$ Asem Ravshanbekovna Alibekova ${ }^{1}$ \\ 1 The Karaganda State University of the name of academician E.A.Buketov, Karaganda, Kazakhstan \\ Correspondence: Nazgul Kadyralievna Tanasheva, The Karaganda State University of the name of academician \\ E.A.Buketov, 100028 Karaganda, Kazakhstan. Tel: 73-877-5948-3532. E-mail: nazgulya_tans@mail.ru
}

Received: October 15, 2014

Accepted: October 25, $2014 \quad$ Online Published: January 11, 2015

doi:10.5539/mas.v9n2p215

URL: http://dx.doi.org/10.5539/mas.v9n2p215

\begin{abstract}
The paper presents the results of experimental studies on determination of the drag and lift force of a rotating cylinder with a porous surface within the airflow velocity range from 5 to $13 \mathrm{~m} / \mathrm{s}(\operatorname{Re}=40,000-105,000)$ at a constant rotation of the cylinder around its axis. There are also presented the results of studies of drag and lift force of a single rotating cylinder within the range of the cylinder revolutions number around its axis from 400 to $1,400 \mathrm{rev} / \mathrm{min}$ at a constant air flow rate. It is shown that the drag coefficient and the lift force coefficient depend on the Reynolds number and the cylinder revolutions number. The dependence of the coefficients of aerodynamic characteristics on the degree of porosity of the surface of the rotating cylinder is established.
\end{abstract}

Keywords: magnus effect, porous surface, re numbers, rotating cylinder

\section{Introduction}

To save fuel and energy resources, to reduce the negative impact on the environment, as well as to supply power to regions, requires the development of harnessing of renewable resources. The Republic of Kazakhstan has huge a potential of renewable energy sources. For example, in most parts of the territory of the Republic of Kazakhstan an average annual wind speed is $3-5 \mathrm{~m} / \mathrm{s}$, therefore the urgent task is to develop a windmill. Of particular interest is the windmill based on rotating cylinders of constant and variable cross-sections that can efficiently operate even at low wind speeds. To optimize the efficiency of the windmill, it is necessary to study the aerodynamic characteristics of the component of a windmill that is of the rotating cylinder. Therefore, this task is relevant both for science and practical use.

Wind energy will play an important role in achieving the energy targets. Both small and industrial sized wind turbine systems have the maturity to be considered economically effective. The small wind turbine market is still developing and could see major growth in the near future (Brusca, 2014).

The analytical review of this topic shows that a wind turbine with revolved cylinders which can operate at low wind speeds is of special interest. The close analogs are two variants of the elements of wind turbines which operate at speeds less than $5 \mathrm{~m} / \mathrm{s}$ : a sail-type wind turbine developed at the Academician Lavrentyev Hydrodynamics Institute of the RAS SB, and a turbine with the use of a cylindrical revolved rotor, i.e. a rotary screw.

Aerodynamic characteristics of cross-flowed fixed single cylinders in an infinite stream has been fairly well studied by S.I. Isataev in his work (Akylbaev, 2003). Effect of cross-section area reduction ratio of flow on the flow pattern of an infinite cylinder on aerodynamic and hydraulic drag are investigated and systematized in the work of Akylbaev Zh.S. (Akylbaev, 2003; Zhangunov, 1987). Aerodynamics of short cylinders, quite often found in power units and installations, is investigated using experimental and theoretical methods in the work of Zhangunov O.N. (Issatayev, 1996).

The flow around a rotating cylinder is a problem in the engineering field, and there are a large number of works reported in literature. Among these results, one of the well known phenomenon is the force called the Magnus effect produced in the direction perpendicular to the direction of the flow. The fluid near the surface of a circular cylinder is dragged along the moving surface due to the viscosity. Accordingly, the pressure in this side decreases and the force is generated in the direction perpendicular to the flow. In connection with this effect, the lift and 
drag of the body in flight having a spin becomes an important problem in the process of developing rotating cylinder.

Swanson has reported, based on his experiments on a rotating cylinder in the transition zone, that the lift acting on the rotating cylinder is not proportional to the increase the rotating speed and it changes depending on the transition of boundary layer (Swanson, 1961). He has also reported that the flow speed increases in the accelerating side, the width of wake becomes smaller, and the Strouhal number of the wake associated with the Karman vortex increases. Furthermore, the Karman vortex is found to disappear for certain spin rate ratios (Tsutahara, 1994).

However, nowadays there are extremely scarce works on the study of aerodynamics of the complicated flow around a single rotating cylinder and a system of the ones which are components of developed by us windmill, the rotating cylinders accompanied by a turbulent flow of the group of interacting vortices. The known works of Bychkov N.M. are concerned with determining of aerodynamic parameters of single rotating cylinders in the airflow (Bychkov, 2005).

The objective of the work is to study the effect of surface porosity on the aerodynamic characteristics of a single rotating cylinder as the main component of a windmill operating at low wind speeds using the Magnus effect (Kussaiynov, 2012; Wojciechowski, 1995).

On the basis of the analysis of existing studies we proposed an experimental investigation of the effects of porosity on the aerodynamic characteristics of a rotating cylinder as component of a windmill, using the Magnus effect.

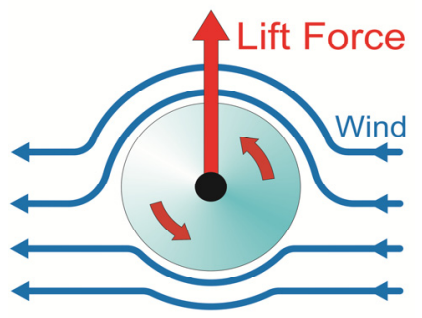

Figure 1. The scheme of the rotating cylinder in the cross-flow

In the rotary motion of the cylinder in the upper air flow, the flow rate and the surface speed are the same, they add up and flow acceleration and increase in speed appear.

At the bottom of the cylinder, the flow rate and the surface speed have opposite directions, they are deducted, there is deceleration and decrease in speed. The appearance of such a difference in speed leads to a transverse pressure difference and the appearance of a transverse lift force, called the Magnus effect, is shown in Figure 1.

Application of Magnus effect at rotating a cylinder in a flow has been known since ancient times (Bolotov, 2004; Akylbaev, \& Turmuhambetov, 1996; Bychkov, 2004), but the possibility of practical use as a driving force for a new generation of windmills for small flow rates has been systematically studied only in recent years.

\section{Phenomenon of the Magnus Effect}

Each of you observed this phenomenon while watching a football match or tennis games. We all know that a soccer ball aimed at the goalposts with a twist moves along a curved locus, a tennis ball flying swiftly over the net with a twist also deflects from the rectilinear locus. At the bottom of these two examples we can see that on bodies flying with rotation in the air flow affects lateral force deflecting from the rectilinear locus, first described in 1853 by a German physicist Heinrich Magnus and named the Magnus effect in his honor. We used this phenomenon in our project to construct a wind turbine. But instead of a ball we have a rotating cylinder which creates a great force of the Magnus effect. In 1924 a German engineer A. Flettner first tested this phenomenon as a rotary engine instead of sails on the ship "Bukau" One of the first wind turbines which used the effect of rotating cylinders was a wind ship of A. Flettner, (Ding, Aidun, 2000; Bychkov, 2004; Chaviaropoulosand, Hansen, 2000). Flettner's wind ship or the Flettner rotor has amazing characteristics that cannot be explained by simple notions of wind pressure, suffice it to say that the use of force on the rotating cylinder should be 10-15 times larger than on a sail with the same visible surface. 

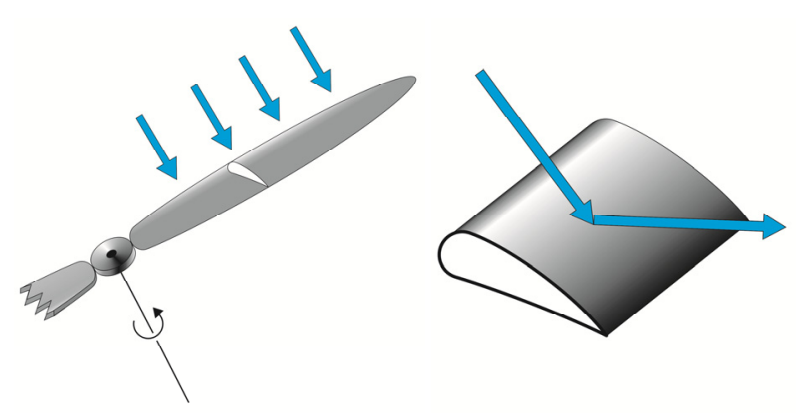

Figure 2. Screw in the air stream (left) and scheme of reflect flow air (right)

At the present time hydrodynamics not only may explain well this seemingly mysterious phenomenon but it also became a planned guide at the discovering of large forceful actions of rotating cylinder. The novelty of the study is that, in contrast to the existing simple helical wind turbines, the blades of which reflect the air flow for small angles is shown in Figure 2, in our wind turbine the cylindrical elements much more efficiently capture the wind flow through the rotation of the cylinders themselves. Due to this fact, the high efficiency of the wind turbine at low wind speeds is ensured is shown in Figure 3.
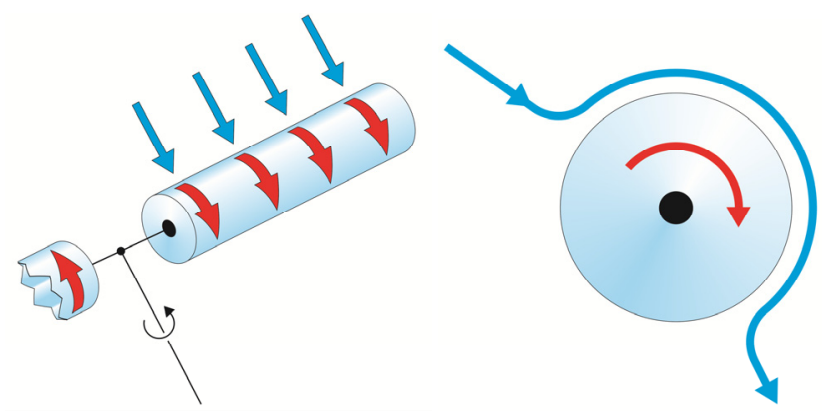

Figure 3. Flow around a rotating cylinder (left) and scheme of capturing the flow of air (right)

\section{Numerical Analysis}

\subsection{Numerical Methods}

The calculation has been performed by the discrete finite volume method in order to solve equations. The velocity field is related by the SIMPLE algorithm with pressure field to obtain the simultaneous solution of the linearized equations by iteration. The governing equations equations are also discretized using Eulerians implicit method, and the time integration technique is used to obtain solutions. The general-purpose heat and fluid software, FLUENT/UNS. Of Fluent Inc. is used for this calculation (Raj, 2000; Himmelskamp, 1947; Aoki, Ito, 2001; Oki, 1998).

\subsection{Boundary Conditions}

Because the fluid to deal with is viscous fluid, it is assumed that the flow does not slip on the cylinder surface, nor the up and down walls.

$$
\text { Namely, } \quad u=0, v=0 .
$$

The velocities and pressure on the inlet and outlet boundary are given as follows:

Inlet:

$$
u=\text { const, } v=0
$$

Turbulent intensity $1 \%$, Outlet: $p=0$.

\subsection{Computational Region and Meshes}

The Object to be analyzed is a two-dimensional circular cylinder. The mesh figure is shown in Figure 4. Setting the diameter of the circular cylinder as $d(10 \mathrm{~cm})$, the areas of analysis are such that the front and the up-down directions are $5 d$, and the back is $15 d$ from the center of circular cylinder. The left hand side is an inlet and the right hand side is an outlet is shown in Figure 5. The up and down sides are the wall. 


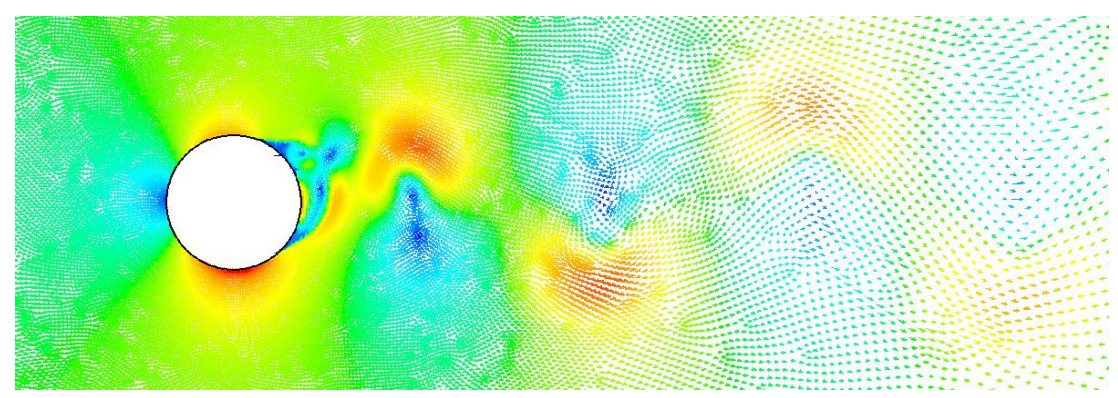

Figure 4. Airflow around the rotating cylinder

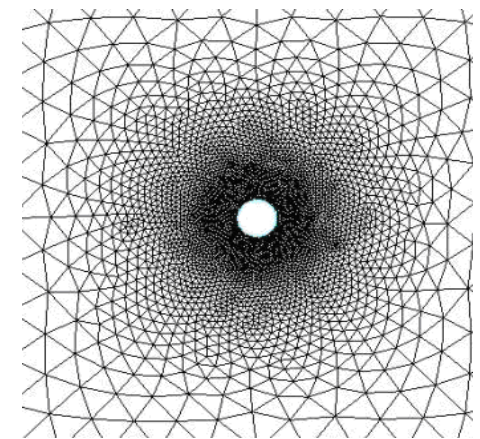

Figure 5. The computational grid

\section{Research Methodology}

The experimental plant was a closed type wind tunnel with an open working section with metal frame hanging on a three-component aerodynamic balance (Sakipova, 2012). The diameter of the working section was $0.5 \mathrm{~m}$, its length was $0.8 \mathrm{~m}$.

Investigations were carried out within the air flow speed range from 5 to $13 \mathrm{~m} / \mathrm{s}(\operatorname{Re}=40,000-105,000)$ at a constant revolutions number of the cylinder around its axis. The drag force and lift force of the rotating cylinder was measured with the three-component aerodynamic balance. The three-component aerodynamic balance makes it possible to measure the drag force and the lift force with a sufficiently high degree of accuracy.

The arrangement of the main components of the plant in the working section of the wind tunnel is shown in Figure 6.

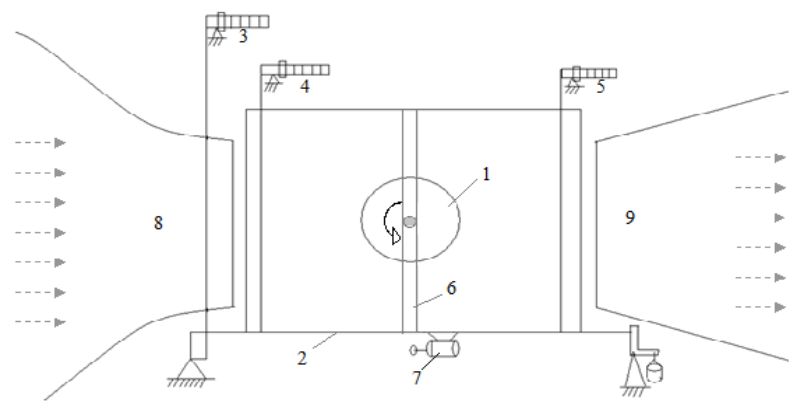

Figure 6. Scheme of the experimental plant for the study of aerodynamic characteristics of the cylinder

Here in Figure 6: 1 - the cylinder under investigation; 2 - a frame for fixing the model with aerodynamic balance; 3 - drag balance; 4, 5 - lift balance; 6 - the rack for mounting cylinders; 7- the engine for rotation of cylinders; 8,9 - the diffuser and contractor of the wind tunnel.

The experimental model was streamlined with a cross air flow, generated in the working section of the wind tunnel is shown in Figure 6. The cylinder was rotated by an electric motor.

The air flow approaching the frontal part of the cylinder, applies a force that is indicated on the balance.

Figure 7 shows a photograph of a cylinder with a porous surface in the working section of a wind tunnel. 


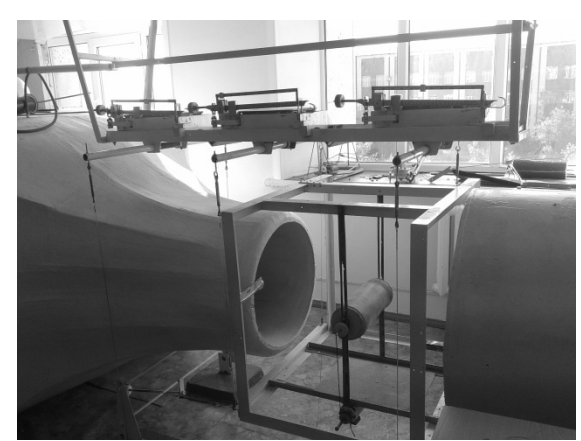

Figure 7. Photo of a cylinder under study installed in the working section of the wind tunnel T-1-M

After measuring, the dependency of the lift force on the rotational frequency of the cylinders with smooth and porous surfaces with a diameter of $50 \mathrm{~mm}$ in the flow at a speed of $U=8 \mathrm{~m} / \mathrm{s}$ and $12 \mathrm{~m} / \mathrm{s}$ have been obtained. We have found that, with the increase in the rotation speed of the cylinder, the lift force increases more than twice and reaches its maximum at a rotation speed of about $1300 \mathrm{rpm}$.

The presence of the porous surfaces leads to an increase in the lift force by $30 \%$ compared to the cylinder with a smooth surface with equal flow conditions. With the increase in the diameter of the cylinder, the lift force increases, for example, at a flow rate of $18 \mathrm{~m} / \mathrm{s}$ the increase in the diameter from 50 to $150 \mathrm{~mm}$ leads to an increase in the lift force of more than 2.5 times. The magnitude of the lift force increases depending on the rotation speed of the cylinder, and this increase occurs only up to a certain maximum magnitude. A further increase in the rotational frequency does not lead to an increase in the lift force. The degree of the porous surfaces does not lead to a significant change in the nature of the dependencies and affects only the numerical values of the drag coefficient, as in the case of the lift force.

Reynolds number and the coefficients of aerodynamic characteristics were calculated as follows:

The formula for determining the Reynolds number:

$$
\operatorname{Re}=\frac{u d}{v}
$$

where $u$ is the velocity of the air flow, approaching the cylinder; $\mathrm{d}$ is the outer diameter of the cylinder under study; $v$ is the kinematic viscosity of air.

The formula for determining the drag coefficient:

$$
C_{x}=\frac{F d}{\frac{\rho u^{2}}{2} S},
$$

where $F d$ is the drag force;

$u$ - is the air flow velocity;

$\rho_{-}$is the air density;

$\mathrm{S}$ - is the area of a middle section of the cylinder under study.

The formula for determining the lift force coefficient:

$$
C_{y}=\frac{F d}{\frac{\rho u^{2}}{2} S},
$$

where $C_{y}$ is the lift force.

We conducted a comprehensive study to determine the dependence of the aerodynamic properties: coefficient of drag and lift coefficient the geometrical parameters and mode parameters that are: Reynolds number and rotation speed of the cylinders.

\section{Results of Research of Aerodynamic Characteristics of a Rotating Cylinder with a Porous Surface}


Tests were performed with of two types rotating cylinders, a diameter of $120 \mathrm{~mm}$ and a length of $330 \mathrm{~mm}$ : Having a smooth surface and a porous surface №1 and № 2. Difference between the two surfaces of the porous cylinder is that the cell sizes of porous surface №1 twice less than sizes of the porous surface №2. The material surface of the cylinder is made of moisture-resistant stainless mesh. The degree of porosity of the cylinder with the porous surface №1 is $25 \%$, and a cylinder with a porous surface № 2 is $50 \%$.

Dependence of drag coefficient $C_{x}$ and lift force coefficient on Re number are shown in figures 8 and 9.

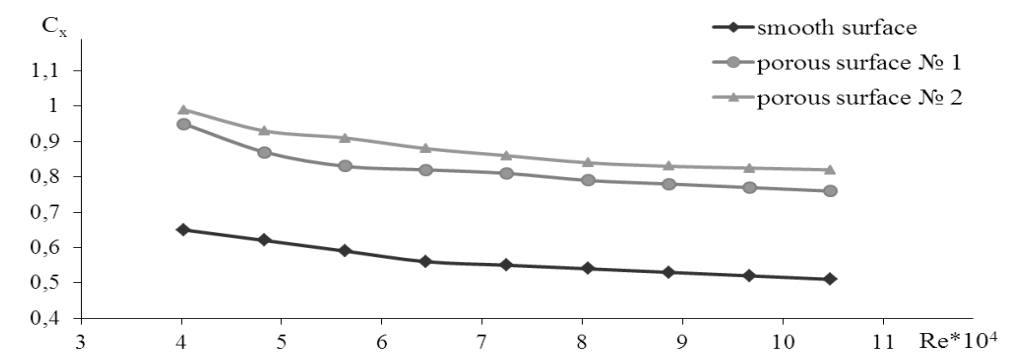

Figure 8. Dependence of $C_{x}$ on the Reynolds number

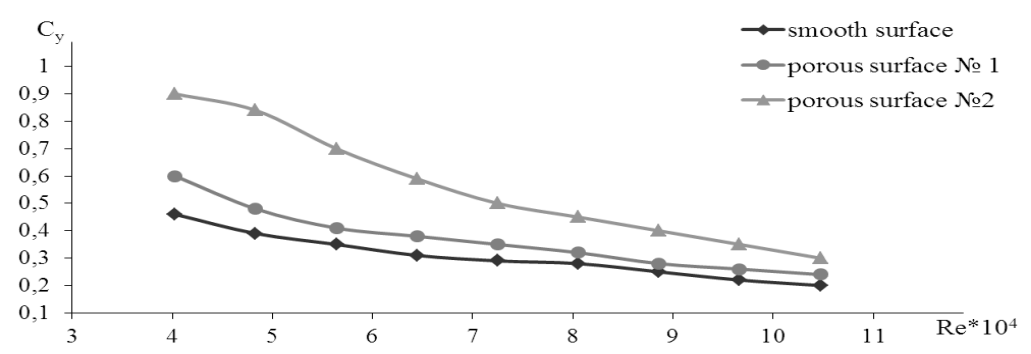

Figure 9. Dependence of the lift force coefficient on the Reynolds number

Figures 8 and 9 show that the drag coefficient and the coefficient of lift force of the rotating cylinder decrease with increasing of flow velocity (Re numbers).

During the experiment, in addition to study of dependency of aerodynamic characteristics coefficients we investigated the dependence of drag coefficient and lift force coefficient on the rotation frequency of the cylinder around its axis at a constant incident flow velocity.

Figure 10 is a graph of dependence of drag coefficient on rotation frequency at a constant velocity of air flow approaching the cylinder.

In Figure 10 you can see that an increase in the rotation frequency of the cylinder results in growth of the drag coefficient. This is due to the fact that, when a stream at a certain velocity flows around a rotating cylinder, vortex flows are formed behind the latter. These vortex flows will interact with particles of the air on the surface of the cylinder at its back and block the incident flow. With an increase in the frequency of rotation of the cylinder, the blocking force of the cylinder against the flow increases. Therefore, the drag of the rotating cylinder increases.

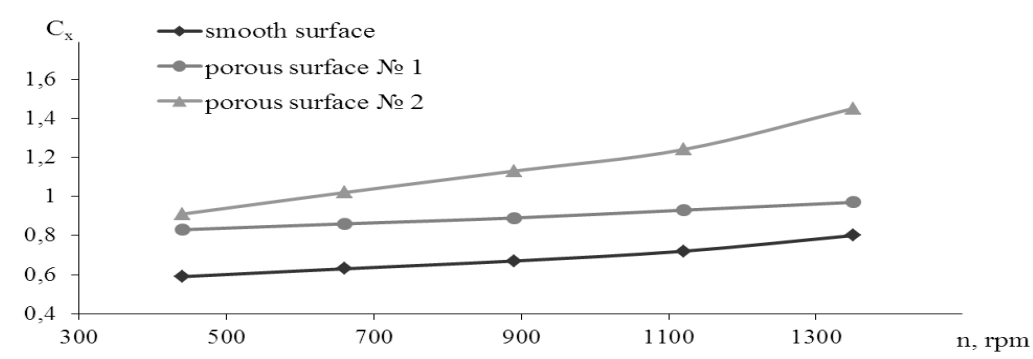

Figure 10. Dependence of the drag coefficient of the rotating cylinder on the rotation frequency

Figure 11 shows the dependence of the lift force coefficient on the frequency of rotation of the cylinder at a 
constant velocity of the cross-flow stream.

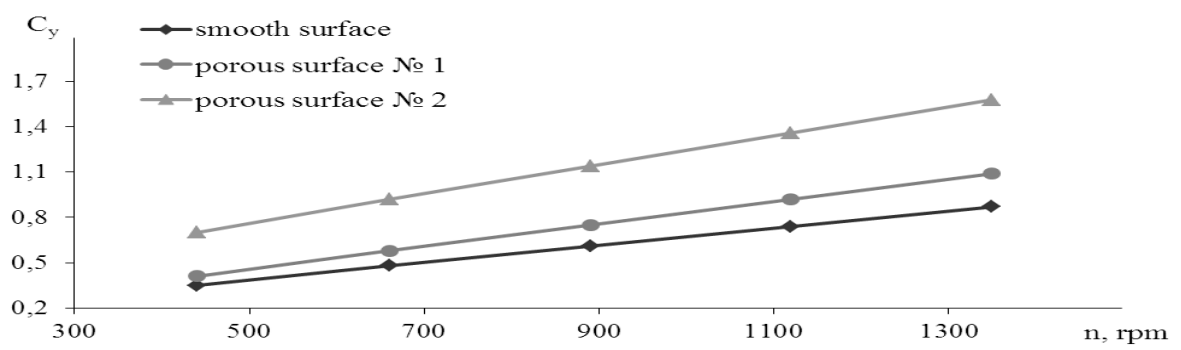

Figure 11. Dependence of the lift force coefficient of a rotating cylinder on the rotation frequency

Figure 11 shows that at a constant velocity of the air flow, the lift force coefficient of the rotating cylinder rises when the rotation frequency of the cylinder increases. This is due to the fact that when the cylinder begins to rotate, it forms a swirling motion around itself. The rotating cylinder entrains the neighboring layers of air; as a result of it, the surrounding air gets, apart from the translational movement, rotation around the cylinder as well. In those places where the velocities of the rotational motion and translational movement are added, the resulting speed exceeds the velocity of the flow, approaching the cylinder. And on the opposite side of the cylinder, the velocities are subtracted and the resulting speed is less than the velocity of the flow away from the cylinder. As a result of it, there is a difference in pressure on the cylinder surface. These differences affect the coefficients of aerodynamic characteristics of a rotating cylinder. The greater the rotation frequency of the cylinders, the more the pressure difference on the cylinder surface. Therefore, the coefficients of aerodynamic characteristics of a rotating cylinder increase.

From the above charts you can see that increase in the porosity of the cylinder surface leads to quantitative growth of coefficients of aerodynamic characteristics of a rotating cylinder. The reason is as follows: when flowing around a rotating cylinder with a porous surface with the air flow, a boundary layer is formed the on the surface of the cylinder that will expand with increase in the degree of porosity.

\section{Conclusions}

On the basis of the data research of the effect of porosity on the aerodynamic characteristics of a rotating cylinder we can draw the following conclusions:

- drag and lift force coefficients of the rotating cylinder as components of a windmill based on Magnus effect depend on the Reynolds number. Increase in the flow velocity (Re numbers), is followed by reduction in the coefficients of lift force and drag by $5-10 \%$.

- Increase in rotation frequency of the cylinder around its axis raises the quantitative values of aerodynamic characteristics of a rotating cylinder, i. e. drag coefficient and the coefficient of lift force.

- aerodynamic characteristics of a rotating cylinder depend on the degree of porosity of the surface of the cylinder. The more the degree of porosity the quantitatively greater drag and lift force coefficients.

Unlike the existing methods, this approach is based on the active capture of airflow by rotating cylindrical elements. The section variability provides an optimal aerodynamic resistance and reasonably high traction for rotating elements. This can be used to create wind turbines of a new generation based on the Magnus effect.

\section{References}

Akylbaev, Z. S. et al. (2003). Aerodynamic features of a rotating cylinder in flow. Industrial heat engineering, 4(25), 279-281.

Akylbaev, Z. S. et al. (2003). Study of lift force of the rotating cylinder in cross-flow of turbulent gas flow. Recent advances in physics and fundamental physical education (pp. 78-83). Almaty: KazSU Publishing House.

Aoki, K., \& Ito, T. (2001). Flow Characteristics around a Rotating Cylinder. Proc. Schl. Eng. Tokai Univ. Ser., 12, 29-34. http://dx.doi.org/10.1007/bf03181548

Bolotov, A. V. et al. (2004). Rotary wind turbines. Collection of scientific works and engineering development of the V Russian exhibition. Dual-use products and technology (Vol. 2, pp. 42-46). Moscow, Russia. Retrieved from http://russianpatents.com/patent/248/2485345.html 
Brusca, S. et al. (2014). Wind Turbine Placement Optimization by means of the Monte Carlo Simulation Method, Modelling and Simulation in Engineering. Retrieved from http://www.hindawi.com/journals/mse/2014/760934/

Bychkov, N. M. (2004). Magnus wind turbine. The results of modeling studies. Thermophysics and Aeromechanics, 11(4), 583-596. Novosibirsk, Russia.

Bychkov, N. M. (2004). Thermophysics and Aeromechanics (pp. 300-567). Novosibirsk, Russia.

Bychkov, N. M. (2005). A wind turbine with Magnus effect. Characteristics of a rotating cylinder, Thermophysics and Aeromechanics, 12(1), 159-175. Retrieved from http://www.sibran.ru/upload/iblock/744/744d9166b640183e4664f1552439f889.pdf

Chaviaropoulosand, P. K., \& Hansen, M. O. (2000). Investigating three-dimensional and rotational effects on wind turbine blades by means of a quasi-3D Navier Stokes solver. Journal of Fluids Engineering, 46, 330-336. http://dx.doi.org/10.1115/1.483261

Ding, E. J., \& Aidun, C. K. (2000). The Dynamics and scaling law for particles suspended in flow with inertia, $J$. Fluid Mech, 25, 317-423. http://dx.doi.org/10.1017/s0022112000001932

Himmelskamp, H. (1947). Profile Investigations on a Rotating Airscrew: MAP Volkenrode Report and

Isatayev, S. I., Akylbaev, Zh. S., \& Turmuhambetov, A. Zh. (1996). Aerohydrodynamics and Heat Exchange of Curvilinear Bodies (pp. 52). Almaty: Gylym.

Issatayev, S. I. et al. (1996). Aerohydrodynamics and heat transfer of curved bodies (pp. 437). Almaty: Gylym.

Kussaiynov, K. et al. (2012). Experimental research of aerodynamics of the system of the revolved cylinders in a turbulent stream. Proceedings of the Seventh International Symposium On Turbulense: Heat and Mass Transfer 7 (pp. 577-580). Palermo. http://dx.doi.org/10.1615/ichmt.2012

Oki, M. et al. (1998). Flow Characteristics around a Circular cylinder with Groove, 4(2), 112-117. http://dx.doi.org/10.1007/bf03181548

Raj, N. V. (2000). An improved semi-empirical model for 3-D post stall effects in horizontal axis wind turbines. Master of Science thesis in Aeronautical and Astronautical Engineering. University of Illinois at Urhana-Champaign: Urhana.

Sakipova, S. E. et al. (2012). Aerodynamics Of The Revolved Cylinders In A Cross Turbulent Stream. Eurasian Physical Technical Journal, 1(17), 39-44. Retrieved from http://www.ksu.kz/files/Faculties/Physical/Eurasian_Journal/2012_17/7.pdf

Swanson, W. M. (1961). The Magnus Effect, ASME, Engineering September, pp. 461. http://dx.doi.org/10.1115/1.3659005

Translation. New York, MAP Volkenrode. http://dx.doi.org/10.2514/1.6564

Tsutahara, M. et al. (1994). Fiows past a Rotating Circular Cylinder by the VIC Method. JSASS, 42(481), 116-124. Retrieved from http://astp.jst.go.jp/modules/search/index.php

Wojciechowski, B. V. (1995). Micro-modular wind power. Institute of Hydrodynamics. Novosibirsk.

Zhangunov, O. N. (1987). Aerodynamics and heat transfer of cylindrical bodies of finite length. Dissert. for the Cand, Sciences degree (pp.144-148). Almaty.

\section{Copyrights}

Copyright for this article is retained by the author(s), with first publication rights granted to the journal.

This is an open-access article distributed under the terms and conditions of the Creative Commons Attribution license (http://creativecommons.org/licenses/by/3.0/). 